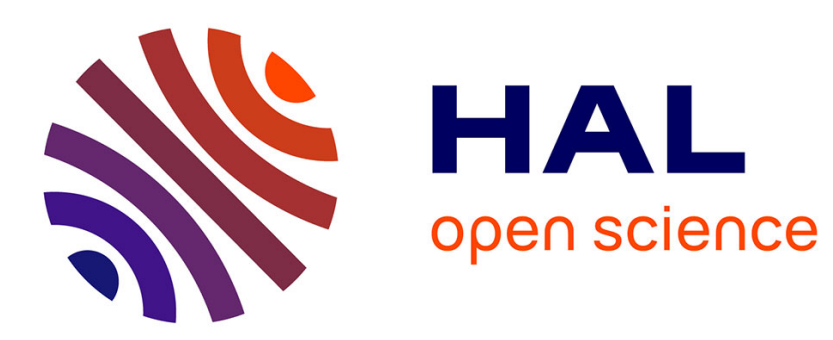

\title{
Interaction of an ultrashort optical pulse with a metallic nanotip: A Green dyadic approach
}

\author{
Arnaud Arbouet, Florent Houdellier, Renaud Marty, Christian Girard
}

\section{To cite this version:}

Arnaud Arbouet, Florent Houdellier, Renaud Marty, Christian Girard. Interaction of an ultrashort optical pulse with a metallic nanotip: A Green dyadic approach. Journal of Applied Physics, 2012, 112 (5), pp.053103. 10.1063/1.4747840 . hal-01742052

\section{HAL Id: hal-01742052 \\ https://hal.science/hal-01742052}

Submitted on 26 Mar 2018

HAL is a multi-disciplinary open access archive for the deposit and dissemination of scientific research documents, whether they are published or not. The documents may come from teaching and research institutions in France or abroad, or from public or private research centers.
L'archive ouverte pluridisciplinaire HAL, est destinée au dépôt et à la diffusion de documents scientifiques de niveau recherche, publiés ou non, émanant des établissements d'enseignement et de recherche français ou étrangers, des laboratoires publics ou privés. 


\section{Interaction of an ultrashort optical pulse with a metallic nanotip: A Green dyadic approach}

Arnaud Arbouet, Florent Houdellier, Renaud Marty, and Christian Girard

Citation: Journal of Applied Physics 112, 053103 (2012); doi: 10.1063/1.4747840

View online: https://doi.org/10.1063/1.4747840

View Table of Contents: http://aip.scitation.org/toc/jap/112/5

Published by the American Institute of Physics

\section{Articles you may be interested in}

Morphology-induced redistribution of surface plasmon modes in two-dimensional crystalline gold platelets

Applied Physics Letters 103, 131112 (2013); 10.1063/1.4823533

Strength of the electric field in apertureless near-field optical microscopy

Journal of Applied Physics 89, 5774 (2001); 10.1063/1.1354655

An ultrafast nanotip electron gun triggered by grating-coupled surface plasmons

Applied Physics Letters 107, 231105 (2015); 10.1063/1.4937121

Tip-based source of femtosecond electron pulses at $30 \mathrm{keV}$

Journal of Applied Physics 115, 094506 (2014); 10.1063/1.4867185

Femtosecond photoelectron point projection microscope

Review of Scientific Instruments 84, 103710 (2013); 10.1063/1.4827035

Sculpting nanometer-sized light landscape with plasmonic nanocolumns

The Journal of Chemical Physics 131, 224707 (2009); 10.1063/1.3271794

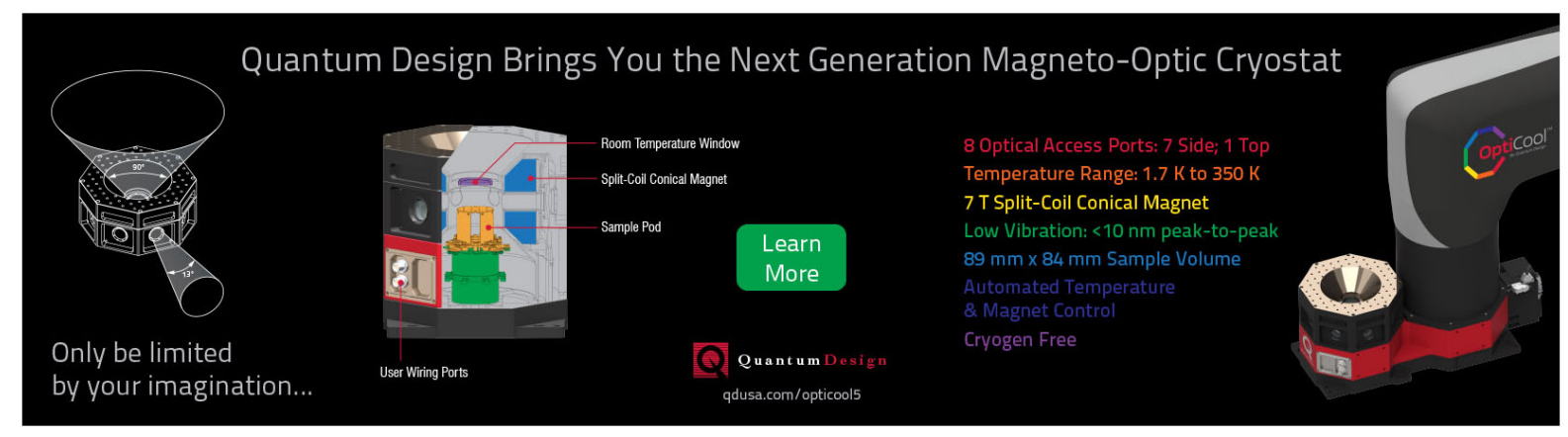




\title{
Interaction of an ultrashort optical pulse with a metallic nanotip: A Green dyadic approach
}

\author{
Arnaud Arbouet, ${ }^{\text {a) }}$ Florent Houdellier, Renaud Marty, and Christian Girard \\ CEMES, CNRS UPR 8011 et Université de Toulouse 29, rue Jeanne Marvig, 31055 Toulouse, France
}

(Received 24 May 2012; accepted 17 July 2012; published online 10 September 2012)

\begin{abstract}
We consider the interaction of an ultrashort optical pulse with a metallic nanotip. In the framework of the Green dyadic method, we calculate the time-dependent electric field in the vicinity of the metallic nano-object. The electric field enhancement at the tip apex, its temporal evolution, and its dependence upon the polarization of the incident electric field are addressed. By comparing the case of tungsten and gold nanotips, we show that significantly larger electric field enhancements can be obtained with the latter and point out different temporal responses. Our description provides the time-dependent spatial distribution of the electric field around metallic nano-objects of arbitrary geometries which is the first step to describe light-matter interaction in recently developed laser-driven femtosecond electron nanosources or recent results in ultrafast nanoplasmonics. (C) 2012 American Institute of Physics. [http://dx.doi.org/10.1063/1.4747840]
\end{abstract}

\section{INTRODUCTION}

The always more demanding research in nanoscience and nanotechnologies has triggered several experimental breakthroughs such as, for instance, the development of near-field probe based investigation techniques that allow to probe matter with ultimate spatial resolution. ${ }^{1}$ In parallel, time-resolved techniques have been developed and it is now possible to observe and control the dynamics of atoms, molecules, and nanosystems on the femtosecond timescale. ${ }^{2}$ Today, new hybrid techniques allowing both spatial and temporal resolutions are actively developed and the quest for ultimate resolutions has brought state of the art experiments in the nanometer-femtosecond domain. For instance, combining femtosecond pump-probe techniques with aperture-type scanning near-field optical microscopy (SNOM) provides simultaneously a spatial resolution of c.a. $100 \mathrm{~nm}$ and a temporal resolution better than $200 \mathrm{fs}^{3-5}$ Ultrafast transmission electron microscopes with nanometer spatial resolution and sub-200 fs time resolution have been developed and brought a wealth of spectacular results. ${ }^{6-8}$ Until now, most of the existing time-resolved electron microscopy and diffraction experiments involved photocathodes in which the electrons are extracted from the electrode by the photoelectric effect. However, in conventional (i.e., not ultrafast) electron microscopy, so-called field emission sources are privileged for demanding applications such as electron interferometry because of their highest brightness and better energy dispersion. In these sources, electrons are tunneling out from the apex of a metallic nanotip under a strong DC bias voltage. Recent experiments demonstrated electron emission from field-emission tips triggered by femtosecond low energy laser pulses. ${ }^{9-11}$ In the latter, different emission regimes have been identified and traced back to analogous experiments performed on atomic systems. ${ }^{12-14}$ However, contrary to their gas phase counterpart, laser-driven ultrafast electron sources based on metallic nanotips do not suffer from averaging on the focal volume as the

a)Electronic mail: arnaud.arbouet@cemes.fr. size of the emitting zone only depends upon the geometry of the emitter. Such ultrafast electron sources allowed to demonstrate the non-dispersive nature of the Aharonov-Bohm effect. ${ }^{15}$ Also, the strong dependence of the interaction between the laser electric field and the tip upon the carrier envelope phase of few femtosecond optical pulses can be exploited to characterize the ultrashort optical pulse. ${ }^{16}$

Beyond fundamental issues related to strong field lasermatter interaction, these studies also raise great expectations as they could lead to new nanometer scale high brightness ultrafast electron sources for time-resolved electron microscopy and interferometry or the development of new microscopy techniques for imaging nanostructures. ${ }^{12,17}$ Laser-driven field emission from both tungsten and gold tips has been demonstrated. ${ }^{9-11}$ The use of tungsten for field emission sources is widespread in conventional transmission electron microscopy (TEM) because of superior emission performances and endurance. However, for laser-driven applications, its damage threshold limits the laser intensities to the multiphoton regime and prevents entering deep into the strong field interaction regime where optical field emission would occur. ${ }^{18,19}$ On the contrary, gold has a larger damage threshold therefore allowing to explore a broader range of electric field strength and interaction regimes. ${ }^{19}$ These current developments in ultrafast electron sources are a strong motivation for the investigation of the material dependent field enhancement and the 3D electric field spatial distribution close to metallic nanotips. In a different context, apertureless or scattering type SNOM (sSNOM) relies on the locally enhanced optical near-field generated near a sharp metal tip upon illumination contrary to aperture-type SNOM in which a subwavelength aperture at the end of an optical fiber collects light from a nanostructured surface. Under CW illumination, it had been shown more than a decade ago that the intensity distribution at the tip apex of scattering type SNOMs is characterized by strong optical field gradients that can be tuned by changing the polarization, wavelength and incidence of the external illumination. ${ }^{20}$ The interpretation of future femtosecond s-SNOM microscopy experiments will undoubtedly require a detailed knowledge of 
the time-dependent electric field close to a metal nanotip. Moreover, recent experiments relying on the interaction of femtosecond optical pulses with noble metal conical tips allowed probing the few-femtosecond electronic dephasing of a local surface plasmon polariton excitation ${ }^{21}$ or even generating $10 \mathrm{~nm}$ spatially and few-femtosecond temporally confined optical excitations. ${ }^{22}$ There is no doubt that the advance of ultrafast nanoplasmonics pushes for the development of theoretical frameworks to compute the femtosecond optical response of nanostructures of arbitrary geometries. ${ }^{21-25}$

Our paper aims at investigating theoretically the interaction of an ultrashort optical pulse with a metallic nanotip in the framework of the Green dyadic method. Two different materials, tungsten and gold, are considered and their electric field enhancements, spatial distributions and temporal evolutions are compared.

\section{TIME-DEPENDENT ELECTRIC FIELDS WITH THE GREEN DYADIC METHOD}

In this section, the Green dyadic method is used to describe the interaction of an ultrashort optical pulse with a nanosystem. This technique is particularly well-suited to describe the optical response of nanostructures of arbitrary geometries, either isolated or electromagnetically coupled or placed in the vicinity of a planar interface..$^{23,26,27}$ For instance, it has been recently shown that this framework can yield the 3D charge distribution inside plasmonic nanostructures. ${ }^{28}$ Other methods such as finite-difference time-domain method (FDTD) ${ }^{29}$ or multiple multipole program (MMP) ${ }^{13}$ could be used to compute time-dependent electric fields.

We consider an arbitrary ultrashort optical pulse $\mathbf{E}_{\text {inc }}(\mathbf{r}, t)$ incident on a nanostructure characterized by a dielectric permittivity $\varepsilon_{m}(\omega)$ placed inside a medium of dielectric constant $\varepsilon_{e n v}$. We calculate the total time-dependent electric field $\mathbf{E}(\mathbf{r}, t)$ arising from the superposition of the incident electric field $\mathbf{E}_{i n c}(\mathbf{r}, t)$ and the optical response of the illuminated nanostructure, i.e., the electric fields reradiated by the nanostructure upon excitation by $\mathbf{E}_{\text {inc }}(\mathbf{r}, t)$. To do so, let us first consider the spectral representation of an arbitrary time-dependent electric field. Any electric field $\mathbf{E}(\mathbf{r}, t)$ can be described equivalently by its Fourier spectrum $\mathbf{E}(\mathbf{r}, \omega)$ defined by

$$
\mathbf{E}(\mathbf{r}, \omega)=\frac{1}{2 \pi} \int_{-\infty}^{+\infty} \mathbf{E}(\mathbf{r}, t) e^{i \omega t} d t .
$$

From Maxwell's equation, one can show that the positiondependent Fourier spectrum of the total electric field $\mathbf{E}(\mathbf{r}, \omega)$ at a given angular frequency $\omega$ obeys the following Helmholtz wave equation:

$$
\Delta \mathbf{E}(\mathbf{r}, \omega)+\mathrm{k}^{2} \mathbf{E}(\mathbf{r}, \omega)=-4 \pi\left[\mathrm{k}_{0}^{2} \mathbf{I}+\frac{1}{\varepsilon_{e n v}} \nabla \nabla\right] \cdot \mathbf{P}(\mathbf{r}, \omega),
$$

where $\mathbf{I}$ is the identity operator, $\mathbf{P}(\mathbf{r}, \omega)$ represents the electric polarization induced inside the nanostructure, $k_{0}=\omega / c$ and $k=\sqrt{\varepsilon_{e n v}} k_{0}$. In the framework of the field-susceptibility theory, the general solution of Helmholtz equation (2) can be written $^{30,31}$

$$
\mathbf{E}(\mathbf{r}, \omega)=\mathbf{E}_{i n c}(\mathbf{r}, \omega)+\int_{V} \mathbf{S}\left(\mathbf{r}, \mathbf{r}^{\prime}, \omega\right) \cdot \mathbf{P}\left(\mathbf{r}^{\prime}, \omega\right) d \mathbf{r}^{\prime} .
$$

In the latter, $V$ is the volume of the nano-tip and $\mathbf{E}_{\text {inc }}(\mathbf{r}, \omega)$ is the Fourier transform of the incident electric field satisfying the homogeneous Helmholtz equation (in the absence of any nanostructure). $\mathbf{S}\left(\mathbf{r}, \mathbf{r}^{\prime}, \omega\right)$ is the field-susceptibility tensor (or Green dyadic function) of the environment. In the framework of the linear response theory, the relationship between the local electric field and the polarization inside the nanostructure is given by

$$
\mathbf{P}\left(\mathbf{r}^{\prime}, \omega\right)=\chi\left(\mathbf{r}^{\prime}, \omega\right) \cdot \mathbf{E}\left(\mathbf{r}^{\prime}, \omega\right) .
$$

Assuming a local response of the material, the susceptibility $\chi\left(\mathbf{r}^{\prime}, \omega\right)$ can be written as

$$
\begin{aligned}
& \chi\left(\mathbf{r}^{\prime}, \omega\right)=\frac{\varepsilon_{m}(\omega)-\varepsilon_{e n v}}{4 \pi} \quad \text { if } \quad r^{\prime} \in V \\
& \chi\left(\mathbf{r}^{\prime}, \omega\right)=0 \quad \text { elsewhere. }
\end{aligned}
$$

The Fourier components $\mathbf{E}(\mathbf{r}, \omega)$ of the total electric field can then be deduced from the self-consistent LippmannSchwinger equation:

$$
\mathbf{E}(\mathbf{r}, \omega)=\mathbf{E}_{i n c}(\mathbf{r}, \omega)+\int_{V} d \mathbf{r}^{\prime} \mathbf{S}\left(\mathbf{r}, \mathbf{r}^{\prime}, \omega\right) \cdot \chi\left(\mathbf{r}^{\prime}, \omega\right) \cdot \mathbf{E}\left(\mathbf{r}^{\prime}, \omega\right) .
$$

This relation can be recast using the generalized propagator $\mathbf{K}\left(\mathbf{r}, \mathbf{r}^{\prime}, \omega\right)$ which directly connects the self-consistent electric field to the incident electric field ${ }^{30,31}$

$$
\mathbf{E}(\mathbf{r}, \omega)=\int_{V} d \mathbf{r}^{\prime} \mathbf{K}\left(\mathbf{r}, \mathbf{r}^{\prime}, \omega\right) \cdot \mathbf{E}_{\text {inc }}\left(\mathbf{r}^{\prime}, \omega\right),
$$

where $\mathbf{K}\left(\mathbf{r}, \mathbf{r}^{\prime}, \omega\right)$ is given by

$$
\mathbf{K}\left(\mathbf{r}, \mathbf{r}^{\prime}, \omega\right)=\mathbf{I} \delta\left(\mathbf{r}-\mathbf{r}^{\prime}\right)+\chi\left(\mathbf{r}^{\prime}, \omega\right) \cdot \mathcal{S}\left(\mathbf{r}, \mathbf{r}^{\prime}, \omega\right) .
$$

Self-consistency is contained in the following Dyson's equation that connects the Green dyadic tensor $\mathcal{S}\left(\mathbf{r}, \mathbf{r}^{\prime}, \omega\right)$ to the field susceptibility tensor $\mathbf{S}\left(\mathbf{r}, \mathbf{r}^{\prime}, \omega\right)$

$$
\begin{aligned}
\mathcal{S}\left(\mathbf{r}, \mathbf{r}^{\prime}, \omega\right)= & \mathbf{S}\left(\mathbf{r}, \mathbf{r}^{\prime}, \omega\right)+\int_{\mathrm{V}} d \mathbf{r}^{\prime \prime} \mathbf{S}\left(\mathbf{r}, \mathbf{r}^{\prime \prime}, \omega\right) \cdot \chi\left(\mathbf{r}^{\prime \prime}, \omega\right) \\
& \cdot \mathcal{S}\left(\mathbf{r}^{\prime \prime}, \mathbf{r}^{\prime}, \omega\right) .
\end{aligned}
$$

The electric field associated with the incident ultrashort optical pulse is described by a superposition of plane waves with a central angular frequency $\omega_{c}$

$$
\mathbf{E}_{i n c}(\mathbf{r}, t)=\Re\left\{\mathbf{E}_{0} \int_{\mathbf{k}} d \mathbf{k} f(\mathbf{k}) e^{-i(\omega t-\mathbf{k} . \mathbf{r})}\right\},
$$

with $k=\sqrt{\varepsilon_{e n v}} \omega / c$ and $\mathbf{E}_{0}$ yields the amplitude and polarization of the electric field. For a monochromatic plane wave $f(\mathbf{k})=\delta\left(\mathbf{k}-\mathbf{k}_{\mathbf{c}}\right)$ in which $k_{c}=\sqrt{\varepsilon_{e n v}} \omega_{c} / c$. We ignore any spatial variation of the incident optical pulse. This approximation is justified because we are interested in the 
time-dependent spatial distribution of the electric field intensity in deeply subwavelength regions that are much smaller than the typical laser focal spot size $(2-10 \mu \mathrm{m})$. This latter assumption could however be given up if needed. The Fourier spectrum of the incident pulse then reads

$$
\begin{aligned}
\mathbf{E}_{i n c}\left(\mathbf{r}, \omega^{\prime}\right) & \\
= & \frac{1}{2 \pi} \int_{-\infty}^{+\infty} \mathbf{E}_{i n c}(\mathbf{r}, t) e^{i \omega^{\prime} t} d t \\
= & \frac{1}{2 \pi} \frac{\mathbf{E}_{0}}{2} \int_{\mathbf{k}} \int_{-\infty}^{+\infty}\left(f(\mathbf{k}) e^{-i \omega t} e^{i \mathbf{k} \cdot \mathbf{r}}+f^{*}(\mathbf{k}) e^{i \omega t} e^{-i \mathbf{k} \cdot \mathbf{r}}\right) e^{i \omega^{\prime} t} d t d \mathbf{k} \\
= & \frac{\mathbf{E}_{0}}{2} \int_{\mathbf{k}} f(\mathbf{k}) e^{i \mathbf{k} \cdot \mathbf{r}} \delta\left(\omega^{\prime}+k c\right) d \mathbf{k} \\
& +\frac{\mathbf{E}_{0}}{2} \int_{\mathbf{k}} f^{*}(\mathbf{k}) e^{-i \mathbf{k} \cdot \mathbf{r}} \delta\left(\omega^{\prime}-k c\right) d \mathbf{k} .
\end{aligned}
$$

The self-consistent electric field Fourier spectrum $\mathbf{E}(\mathbf{r}, \omega)$ in the vicinity of the nanostructure can then be deduced from the latter equation:

$$
\begin{aligned}
\mathbf{E}\left(\mathbf{r}, \omega^{\prime}\right)= & \int_{V} d \mathbf{r}^{\prime} \mathbf{K}\left(\mathbf{r}, \mathbf{r}^{\prime}, \omega^{\prime}\right) \cdot \mathbf{E}_{i n c}\left(\mathbf{r}^{\prime}, \omega^{\prime}\right) \\
= & \frac{\mathbf{E}_{0}}{2}\left(\int_{\mathbf{k}} d \mathbf{k} f(\mathbf{k}) \delta\left(\omega^{\prime}+k c\right) \int_{V} d \mathbf{r}^{\prime} \mathbf{K}\left(\mathbf{r}, \mathbf{r}^{\prime}, \omega^{\prime}\right) e^{i \mathbf{k} . \mathbf{r}^{\prime}}\right. \\
& \left.+\int_{\mathbf{k}} d \mathbf{k} f^{*}(\mathbf{k}) \delta\left(\omega^{\prime}-k c\right) \int_{V} d \mathbf{r}^{\prime} \mathbf{K}\left(\mathbf{r}, \mathbf{r}^{\prime}, \omega^{\prime}\right) e^{-i \mathbf{k} . \mathbf{r}^{\prime}}\right) .
\end{aligned}
$$

Finally, the inverse Fourier transform provides the total electric field

$$
\begin{aligned}
\mathbf{E}(\mathbf{r}, t) & =\int_{-\infty}^{+\infty} \mathbf{E}\left(\mathbf{r}, \omega^{\prime}\right) e^{-i \omega^{\prime} t} d \omega^{\prime} \\
& =\Re\left\{\mathbf{E}_{0} \int_{\mathbf{k}} d \mathbf{k} f(\mathbf{k}) \int_{V} d \mathbf{r}^{\prime} \mathbf{K}\left(\mathbf{r}, \mathbf{r}^{\prime}, k c\right) e^{-i\left(\omega t-\mathbf{k} \cdot \mathbf{r}^{\prime}\right)}\right\} .
\end{aligned}
$$

In the latter equation, $\omega=k c / \sqrt{\varepsilon_{\text {env }}}$ and we have taken into account the fact that $\mathbf{K}\left(\mathbf{r}, \mathbf{r}^{\prime},-k c\right)=\mathbf{K}^{*}\left(\mathbf{r}, \mathbf{r}^{\prime}, k c\right)$ required to preserve causality. ${ }^{32}$

By comparing Eq. (12) with Eq. (10), one can notice that the total electric field in the vicinity of a nano-object can be computed from the incident electric field in a three-step procedure. First, the incident electric field is Fourier-transformed into a superposition of monochromatic plane waves with a corresponding weight given by $f(\mathbf{k})$. Then, the optical response of the illuminated nanostructure to an excitation by a single monochromatic plane wave is computed using the generalized propagator. Finally, the total electric field is reconstructed from the individual contributions of the different monochromatic plane waves of the incident optical pulse.

\section{INTERACTION OF A FEMTOSECOND OPTICAL PULSE WITH A METALLIC NANOTIP}

We focus now on the situation depicted in Figure 1(a). An ultrashort optical pulse propagating in vacuum $\left(\varepsilon_{e n v}=1\right)$ is incident along (OZ) on a metallic nanotip having its sym- a)

b)

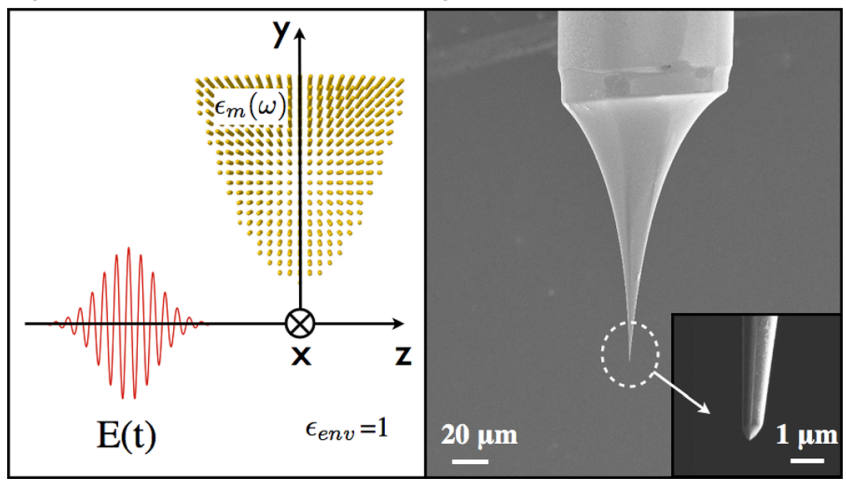

FIG. 1. (a) An ultrafast optical pulse is incident along (OZ) on a metallic nanotip. The nanotip symmetry axis is (OY). (b) SEM image of a tungsten nanotip. Inset: close-up of the tip apex.

metry axis along (OY). Figure 1(b) is an example of a field emission tip for electron microscopy classically fabricated by electrochemical etching of a tungsten nanowire. As shown in the inset of Figure 1(b), such nanotips have several facets oriented along different crystallographic orientations (W[310] being privileged for TEM). In the following, we neglect this structuration as the characteristic lengths are smaller than the optical wavelength and can vary from tip to tip. We then assume that the apex can be represented by a paraboloid with a radius of curvature $p=30 \mathrm{~nm}$ which is typical for conventional tungsten nanotips. We use the bulk dielectric constant of gold and tungsten $\varepsilon_{m}(\omega)$, respectively, taken from Johnson and Christy ${ }^{33}$ and Palik. ${ }^{34}$

As detailed in Sec. II, the incident ultrashort optical pulse is represented by a superposition of monochromatic plane waves with wavevectors directed along (OZ). This summation can be equivalently performed on the angular frequencies $\omega$

$$
\mathbf{E}_{\text {inc }}(\mathbf{r}, t)=\int \mathbf{E}_{0} f(\omega) e^{-i(\mathbf{k} \cdot \mathbf{r}-\omega t)} d \omega=\int \mathbf{E}_{0} f(\omega) e^{-i\left(k_{z} z-\omega t\right)} d \omega,
$$

in which $k_{z}=\omega / c$. Two different linear polarizations are considered, $\mathbf{E}_{0}$ being aligned either along (OX) or (OY). The optical pulse has a gaussian profile with a FWHM of $10 \mathrm{fs}$ and a central wavelength $\lambda_{c}=800 \mathrm{~nm}$. Assuming that the electric field is initially maximum in $z=0$, we can write

$$
\mathbf{E}_{i n c}(\mathbf{r}=0, t)=\Re\left\{\mathbf{E}_{0} e^{-a t^{2}-i \omega_{c} t}\right\} .
$$

The Fourier transform of the incident electric field can therefore be written

$$
\mathbf{E}_{i n c}(\mathbf{r}=0, \omega)=\mathbf{E}_{0} \frac{1}{\sqrt{4 \pi a}} e^{-\frac{\left(\omega-\omega_{c}\right)^{2}}{4 a}}
$$

Figure 2(a) shows an example of a $10 \mathrm{fs}$ optical pulse with a central wavelength $\lambda_{c}=800 \mathrm{~nm}$. The spectrum shown in Figure 2(b) has a full width at half maximum of c.a. $100 \mathrm{~nm}$.

Our procedure is as follows. First, the spectrum of the optical pulse is sampled on a set of frequencies $\left\{\omega_{i}\right\}$. Typically, 100 different frequencies spanning a $1 \mathrm{eV}$ range 
a)

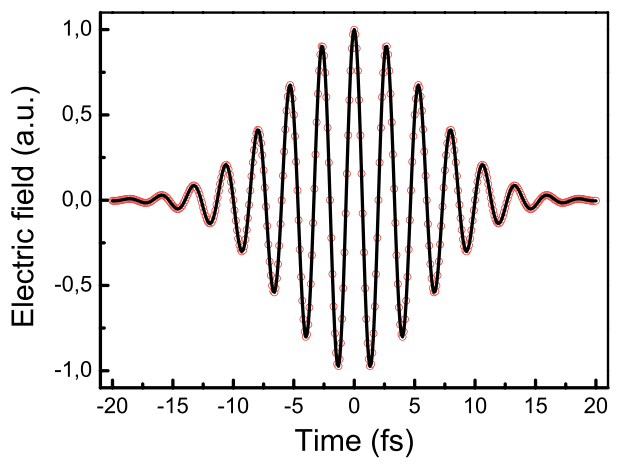

b)

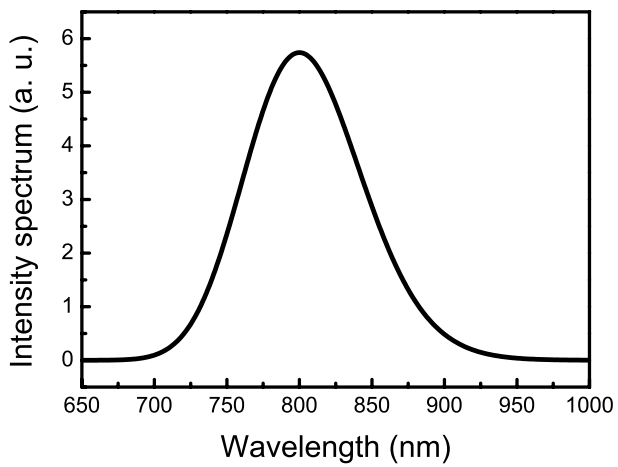

FIG. 2. (a) Initial (solid line) and reconstructed (dots) electric field of a $10 \mathrm{fs}$ optical pulse centered at $800 \mathrm{~nm}$. (b) Corresponding intensity spectrum. centered at $1.55 \mathrm{eV}$ (i.e., $0.01 \mathrm{eV}$ energy step) are used. As illustrated in Figure 2(a), care was taken that the electric field reconstructed from the sampled spectrum faithfully reproduces the initial pulse. Artificial periodic replica due to sampling is not an issue as they appear at larger time delays (several picoseconds for this sampling). In a second step, the total electric field obtained upon excitation of the nanostructure by a monochromatic plane wave is calculated using Eq. (7) for each frequency $\omega_{i}$. The total time-dependent electric field $\mathbf{E}(\mathbf{r}, t)$ is finally deduced from Eq. (12). The robustness of the final results with respect to the sampling parameters has been systematically checked. In the following, we are interested in the time-dependent electric field amplitude and optical near-field intensity $I(\mathbf{r}, t)$, the latter being defined as

$$
I(\mathbf{r}, t)=|\mathbf{E}(\mathbf{r}, t)|^{2} .
$$

First, we compute the time dependent electric field intensity at the tip apex of a gold tip for different polarizations. In our simulations, the incident ultrashort optical pulse has its maximum amplitude at the tip apex at $\mathrm{t}=0 \mathrm{fs}$. As shown Figure 1(a), the metallic nanotips have been meshed on a cubic lattice composed of 4000 points. Figure 3 shows a sequence of $2 \mu \mathrm{m} \times 2 \mu \mathrm{m}$ intensity maps computed in the (OXZ) planes located $20 \mathrm{~nm}$ below the tip apex at different times $t$. Two cases have been considered: the incident electric field is polarized perpendicularly to the tip axis for Figures 3(a)3 (c), whereas it is parallel for (d)-(f). On the (OXZ) maps presented in the remainder of the paper, the tip is at the

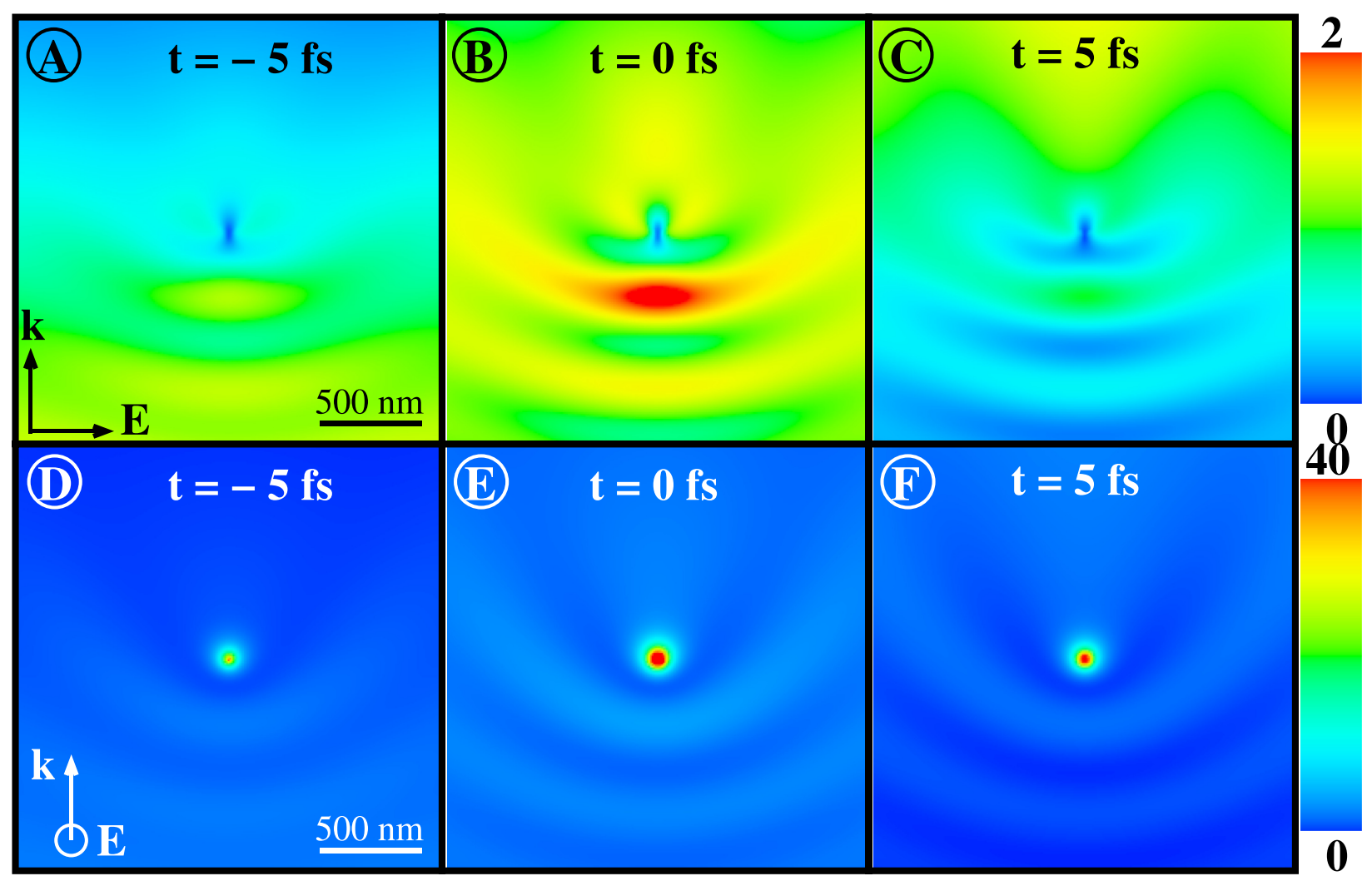

FIG. 3. $2 \mu \mathrm{m} \times 2 \mu \mathrm{m}$ electric field intensity maps computed in the (OXZ) plane located $20 \mathrm{~nm}$ below the apex of a gold tip at different times $t$. The incident electric field is polarized along (OX) in (a)-(c) and along (OY) in (d)-(f). 


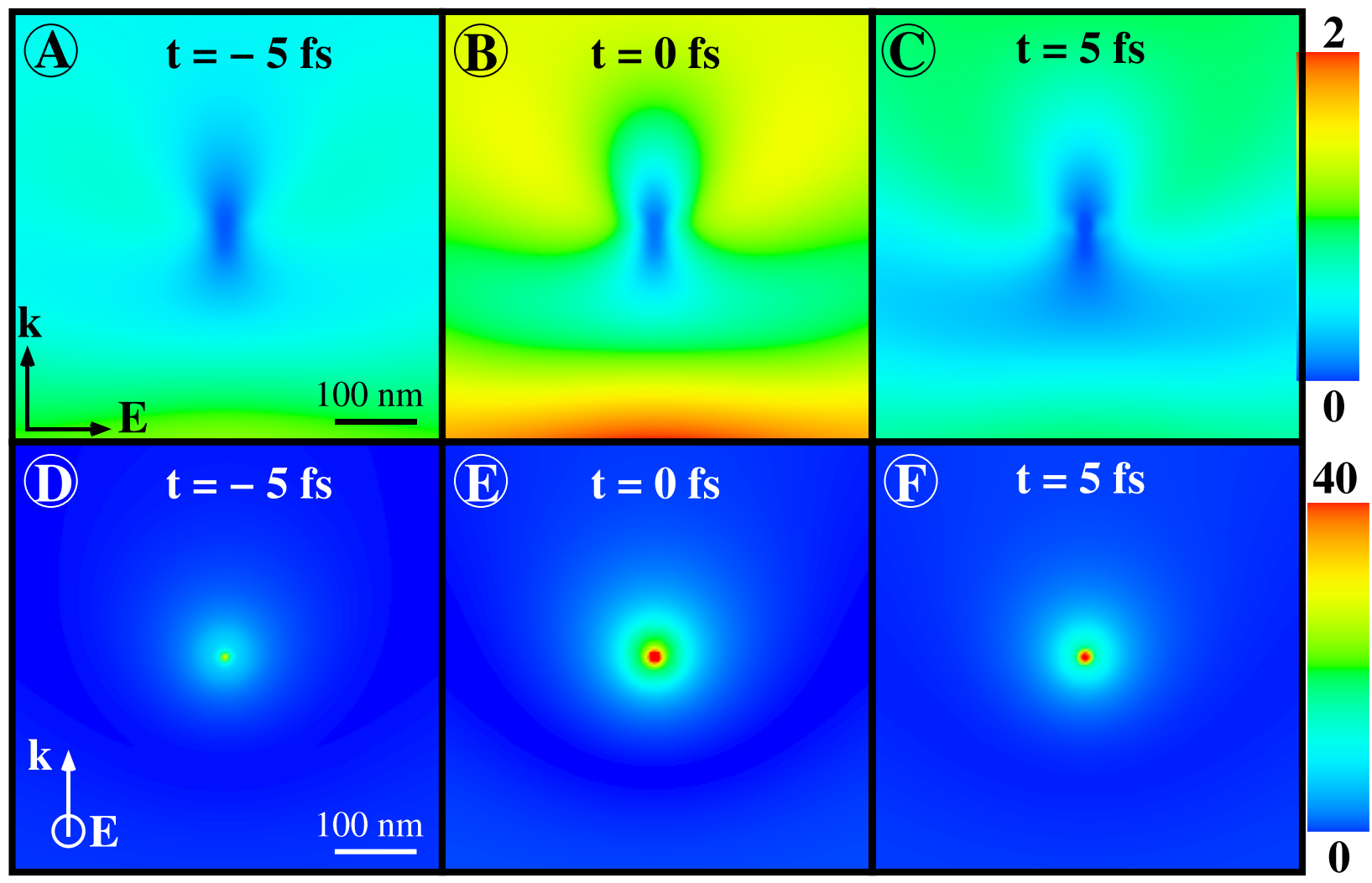

FIG. 4. $500 \mathrm{~nm} \times 500 \mathrm{~nm}$ electric field intensity maps computed in the (OXZ) plane located $20 \mathrm{~nm}$ below the apex of a gold tip at different times $t$. The incident electric field is polarized along (OX) in (a)-(c) and along (OY) in (d)-(f).

center of the maps and the femtosecond optical pulse is traveling from bottom to top. A clear difference is evidenced between the two polarizations: electric fields parallel to the tip axis give rise to 20 times larger enhancements and more localized high-intensity regions. Fringes due to interferences between the incident and scattered electric fields are clearly visible on the side of the incoming beam..$^{20,35}$

Figure 4 is a magnification of the tip apex region. The intensity pattern is similar to the near-field intensity pattern generated by an oscillating dipole. In particular, we observe a dip in intensity in a direction perpendicular to the incident electric field in Figures 4(a)-4(c). On the contrary, in Figures 4(d)-4(f)), a large electric field enhancement is apparent along the electric field polarization (OY).

Figures 5(a) and 5(b) show intensity maps in the (OXY) and (OYZ) planes for an incident electric field polarized along (OY). The maps computed in the (OXY) plane are symmetrical with respect to the nanotip axis (OY) as expected. On the contrary, due to propagation of the optical pulse along the (OZ) axis, this symmetry is lost in the (OYZ) plane and stronger electric fields are found in the direction of propagation. Movies showing the temporal evolution of the electric field close to the nanotip apex are available as supplementary information. ${ }^{37}$

\section{COMPARISON OF GOLD AND TUNGSTEN NANOTIPS: ELECTRIC FIELD ENHANCEMENT AND TEMPORAL RESPONSE}

In the following, we compare the ultrafast optical response of gold and tungsten nanotips. Figures 5(c) and 5(d) show the electric field intensity maps computed on a tungsten tip for an incident electric field polarized along (OY). Both the geometry of the tip and illumination conditions (intensity and polarization) have been kept exactly identical as in the corresponding data for gold shown in Figures 5(a) and 5(b). Our results show that the gold nanotip yields approximately

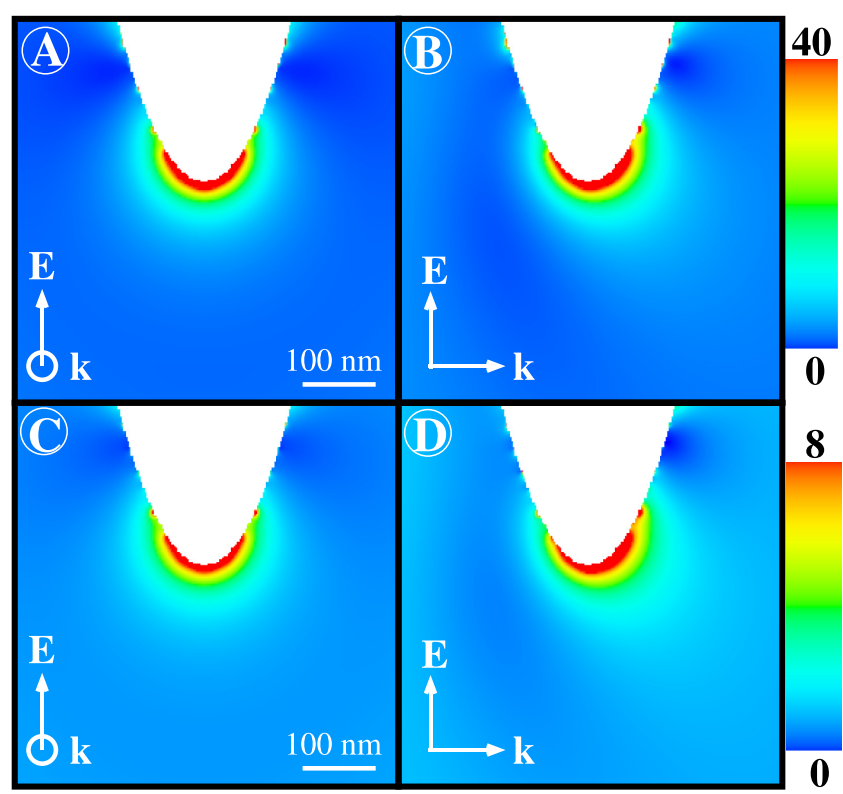

FIG. 5. (a) $500 \mathrm{~nm} \times 500 \mathrm{~nm}$ electric field intensity maps computed in the (OXY) plane at $t=0$ fs. (b) Same as (a) in the (OYZ) plane. (c) and (d) show corresponding data in the case of a tungsten tip. The incident electric field is polarized along (OY). 
5 times larger electric field intensities in the apex region than its tungsten counterpart. This larger enhancement of the electric field is due to the more pronounced free electron character of gold compared to tungsten: electrons can more easily respond to the electromagnetic excitation and the excited surface plasmons yield more intense optical near-fields. This difference has been experimentally confirmed in the context of laser-driven field emission from metallic nanotips: the larger electric fields at the apex of gold nanotips allow to identify the transition from the multiphoton to the optical field emission regimes. ${ }^{19}$ This can have important consequences since identical illumination conditions can lead in these two materials to different interaction regimes. Indeed, electron emission from metallic nanotips can be obtained following different mechanisms that can be directly traced back from the well-established literature of atomic physics and attosecond science. ${ }^{36}$ These different emission regimes can be discriminated using the Keldysh parameter $\gamma$ that gives the ratio between the electron tunneling time and the laser optical period. In atomic physics, $\gamma=\sqrt{I_{p} /\left(2 U_{p}\right)}$ in which $I_{p}$ is the ionization potential and $U_{p}$ is the ponderomotive energy. In the case of metallic tips submitted to an additional DC electric field, $I_{p}$ has to be replaced by the effective work function $W_{\text {eff }}$ of the metal that takes into account the lowering of the potential barrier by the Schottky effect due to the applied DC voltage. Therefore, both DC field and laser electric field will have an impact on the Keldysh parameter and the interaction regime. ${ }^{13}$ Under the same illumination condition, our previous results show that gold nanotips generate a 5 times larger laser electric field intensity at the apex compared to tungsten tips. As a consequence, the corresponding Keldysh parameter of a gold nanotip is expected to be 2 to 3 times smaller in this case than for a tungsten tip. In consequence, gold nanotips should be privileged for investigations of strong field effects. ${ }^{12,19}$ From a practical point of view, the typical electric field strength required for field emission is of the order of $1 \mathrm{GV} / \mathrm{m}$. The field enhancements existing at the apex of metallic nanotips allow to obtain electron emission at much lower laser intensities than needed in the case of atoms or molecules. For instance, a $80 \mathrm{MHz}$ train of $10 \mathrm{fs}$ pulses with an average power of only $10 \mathrm{~mW}$ focused on a few $\mu \mathrm{m}$ spot yields a peak intensity (cycle averaged) in the $10^{11} \mathrm{~W} / \mathrm{cm}^{2}$ range and electric fields of the order of $1 \mathrm{GV} / \mathrm{m}$.

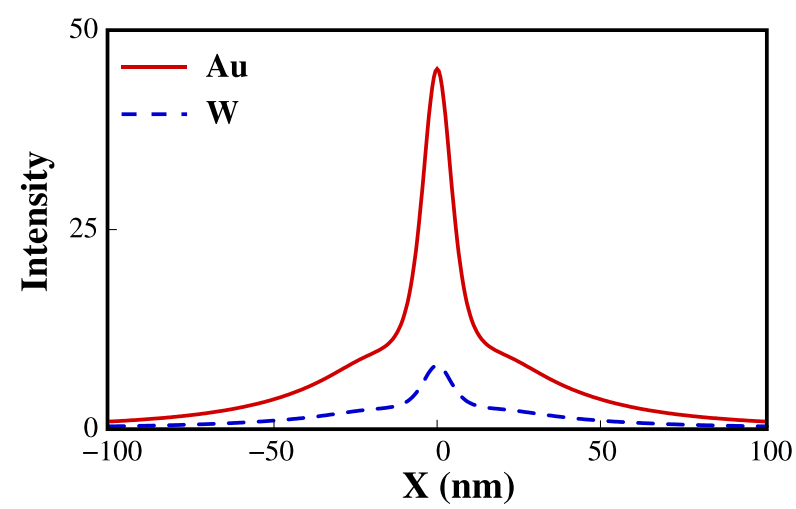

FIG. 6. (a) Electric field intensity profiles along (OX) for gold and tungsten nanotips. $t=0 \mathrm{fs}, Y_{o b s}=-20 \mathrm{~nm}$, and $Z_{o b s}=0 \mathrm{~nm}$. The incident electric field is polarized along the tip axis (OY).
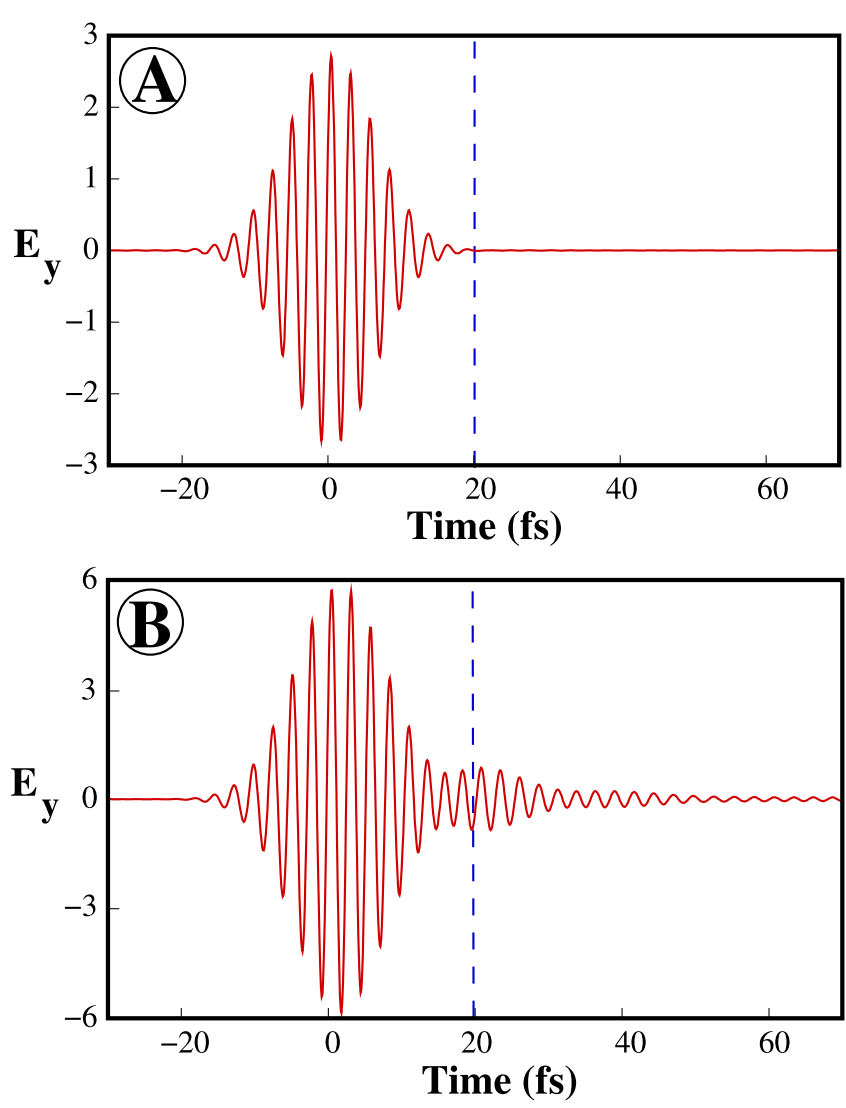

FIG. 7. (a) Time dependent electric field amplitude $E_{Y}$ for a tungsten tip. $X_{o b s}=0 \mathrm{~nm}, Y_{o b s}=-20 \mathrm{~nm}$, and $Z_{o b s}=0 \mathrm{~nm}$. (b) Same for a gold tip. The incident electric field is polarized along the tip axis (OY).

To go further, we investigate the electric field intensity profiles for both materials. Figure 6 shows that the extension of the high-intensity region is not significantly modified when considering gold tips instead of tungsten ones, the full width at half maximum after normalization of the data being the same. This behavior confirms that for metals the topography of the electric field is mainly governed by the morphology of the nanotip.

Finally, we have calculated the time-dependent electric fields close to the apex of tungsten and gold nanotips. In the case of tungsten (Figure 7(a)), we can notice that the electric field faithfully reproduces the excitation pulse presented in Figure 2(a). This is due to the large bandwidth of the optical response of the metal. Figure 7(b) shows a different response in the case of a gold nanotip in which the electric field at the apex survives several tens of femtoseconds after the passage of the ultrashort optical pulse. This is a consequence of the excitation of surface plasmons at the surface of the metallic nano-object with a damping time larger than the excitation pulse duration. Similar results have been obtained at the hot spots of a plasmonic composite nanostructure ${ }^{23}$ or on silver films. $^{24}$

\section{CONCLUSION}

In this paper, we have adapted the standard Green dyadic method to compute the time-dependent electric field at the apex of a metallic nanotip. The distribution of the optical 
electric field generated in this region has been thoroughly investigated. We have shown that gold nanotips provide 5 times larger electric field intensities than tungsten ones and should therefore be used for experiments in the strong field interaction regime. In the case of gold tips, we have pointed out a difference in temporal response of the metallic tip due to the excitation of surface plasmon excitations. The presented framework could address the wavelength-dependent influence of these surface plasmon resonances on the ultrafast optical response of a nanostructure or describe the evolution of the latter upon illumination by arbitrary optical waveforms. Our method provides a complete description of the optical response of structures of arbitrary geometries in both space and time. It could be used for the modeling of electron trajectories in intense ultrashort optical pulses or the interpretation of ultrafast near-field optical microscopy experiments or recent results in ultrafast nanoplasmonics. ${ }^{25}$ For few cycle optical pulses, carrier-envelope phase effects are expected to play a major role and should be considered in details. A straightforward extension of this work could deal with these effects.

\section{ACKNOWLEDGMENTS}

This work was supported by the computing facility center CALMIP of Paul Sabatier University.

${ }^{1}$ D. W. Pohl, W. Denk, and M. Lanz, Appl. Phys. Lett. 44, 651 (1984).

${ }^{2}$ M. Shapiro and P. Brumer, Principles of the Quantum Control of Molecular Processes (Wiley, New York, 2003).

${ }^{3}$ B. A. Nechay, U. Siegner, M. Achermann, H. Bielefeldt, and U. Keller, Rev. Sci. Instrum. 70, 2758 (1999).

${ }^{4}$ H. Okamoto and K. Imura, Prog. Surf. Sci. 84, 199 (2009).

${ }^{5} \mathrm{Z}$. Li, S. Yue, J. Chen, and Q. Gong, Opt. Express 18, 14232 (2010).

${ }^{6}$ V. A. Lobastov, R. Srinivasan, and A. H. Zewail, Proc. Natl. Acad. Sci. U.S.A. 102, 7069 (2005).

${ }^{7}$ W. E. King, M. R. Armstrong, O. Bostanjoglo, and B. W. Reed, Science of Microscopy, edited by P. W. Hawkes and J. C. H. Spence (Springer, 2007), p. 406

${ }^{8}$ A. H. Zewail and J. M. Thomas, $4 D$ Electron Microscopy: Imaging in Space and Time (Imperial College, 2009).

${ }^{9}$ P. Hommelhoff, C. Kealhofer, and M. A. Kasevich, Phys. Rev. Lett. 97, 247402 (2006)

${ }^{10}$ P. Hommelhoff, Y. Sortais, A. Aghajani-Talesh, and M. A. Kasevich, Phys. Rev. Lett. 96, 077401 (2006).
${ }^{11}$ B. Barwick, C. Corder, J. Strohaber, N. Chandler-Smith, C. Uiterwaal, and H. Batelaan, New J. Phys. 9, 142 (2007).

${ }^{12}$ C. Ropers, D. R. Solli, C. P. Schulz, C. Lienau, and T. Elsaesser, Phys. Rev. Lett. 98, 043907 (2007).

${ }^{13}$ H. Yanagisawa, C. Hafner, P. Doná, M. Klöckner, D. Leuenberger, T. Greber, J. Osterwalder, and M. Hengsberger, Phys. Rev. B 81, 115429 (2010).

${ }^{14} \mathrm{H}$. Yanagisawa, M. Hengsberger, D. Leuenberger, M. Klöckner, C. Hafner, T. Greber, and J. Osterwalder, Phys. Rev. Lett. 107, 087601 (2011).

${ }^{15}$ A. Caprez, B. Barwick, and H. Batelaan, Phys. Rev. Lett. 99, 210401 (2007).

${ }^{16}$ M. Krüger, M. Schenk, and P. Hommelhoff, Nature 475, 78 (2011).

${ }^{17}$ A. Arbouet and F. Houdellier, patent pending PCT 125140 (February 2012).

${ }^{18}$ S. A. Hilbert et al., J. Phys. B 42, 141001 (2009).

${ }^{19}$ R. Bormann, M. Gulde, A. Weismann, S. V. Yalunin, and C. Ropers, Phys. Rev. Lett. 105, 147601 (2010).

${ }^{20}$ O. J. F. Martin and C. Girard, Appl. Phys. Lett. 70, 705 (1997).

${ }^{21}$ A. Anderson, K. S. Deryckx, X. G. Xu, G. Steinmeyer, and M. B. Raschke, Nano Lett. 10, 2519 (2010).

${ }^{22}$ S. Berweger, J. M. Atkin, X. G. Xu, R. L. Olmon, and M. B. Raschke, Nano Lett. 11, 4309 (2011).

${ }^{23}$ M. I. Stockman, New J. Phys. 10, 025031 (2008).

${ }^{24}$ P. Dombi, S. E. Irvine, P. Rácz, M. Lenner, N. Kroó, G. Farkas, A. Mitrofanov, A. Baltuska, T. Fuji, F. Krausz, and A. Y. Elezzabi, Opt. Express 18, 24206 (2010).

${ }^{25}$ C. Rewitz, T. Keitzl, P. Tuchscherer, J.-S. Huang, P. Geisler, G. Razinskas, B. Hecht, and T. Brixner, Nano Lett. 12(1), 45 (2012).

${ }^{26}$ C. Girard and A. Dereux, Rep. Prog. Phys. 59, 657 (1996).

${ }^{27}$ S. Tripathy, R. Marty, V. K. Lin, S. L. Teo, E. Ye, A. Arbouet, L. Saviot, C. Girard, M. Y. Han, and A. Mlayah, Nano Lett. 11(2), 431 (2011).

${ }^{28}$ R. Marty, G. Baffou, A. Arbouet, C. Girard, and R. Quidant, Opt. Express 18, 3035 (2010)

${ }^{29}$ S. E. Irvine and A. Y. Elezzabi, Phys. Rev. A 73, 013815 (2006).

${ }^{30}$ O. J. F. Martin, C. Girard, and A. Dereux, Phys. Rev. Lett. 74, 526 (1995).

${ }^{31}$ C. Girard, Rep. Prog. Phys. 68, 1883 (2005).

${ }^{32}$ L. D. Landau and E. M. Lifshitz, Electrodynamics of Continuous Media (Pergamon, London, 1960).

${ }^{33}$ P. Johnson and R. Christy, Phys. Rev. B 6, 4370 (1972).

${ }^{34}$ E. Palik, Handbook of Optical Constants of Solids (5 Volume Set) (Academic, 1997).

${ }^{35}$ Y. C. Martin, H. F. Hamann, and H. K. Wickramasinghe, J. Appl. Phys. 89, 5774 (2001).

${ }^{36}$ M. Krüger, M. Schenk, M. Förster, and P. Hommelhoff, J. Phys. B 45, 074006 (2012).

${ }^{37}$ See supplementary material at http://dx.doi.org/10.1063/1.4747840 for movies of the temporal evolution of the optical near-field intensity. The movies display the optical near-field intensity in a 2 micron $\times 2$ micron region of the plane perpendicular to the nanotip and located $20 \mathrm{~nm}$ below it. The two cases of an incident polarization perpendicular or parallel to the nanotip are shown. 